\title{
On the Use of Digital Image Correlation for the Analysis of the Dynamic Behavior of Materials
}

\author{
François Hild, Amine Bouterf, Pascal Forquin and Stéphane Roux
}

\begin{abstract}
The present chapter is devoted to the analysis of the mechanical behavior of materials subjected to dynamic loadings via digital image correlation (DIC). This measurement technique provides $2 \mathrm{D}$ or $3 \mathrm{D}$ displacement fields that can be evaluated thanks to the use of high-speed cameras. Various declinations of DIC are first presented. Uncertainty quantifications are also discussed. Last, different examples illustrate how DIC can be used to analyze and quantify deformation, damage and fracture mechanisms of brittle and ductile materials.
\end{abstract}

\section{Introduction}

The development of reliable measurement techniques is very important when characterizing heterogeneous mechanical manifestations at various scales. Solids, be they heterogeneous or homogeneous under complex loading, induce deformations whose multiscale analysis is needed to relate them to the underlying microstructure and / or to the type of prescribed loading. From the knowledge of kinematic fields, it is possible to identify global and local material parameters, or validate material models and numerical tools [1].

Different techniques are used to measure displacement and strain fields under dynamic loading conditions. When utilizing optics concepts, and when coupled with mechanics, it is referred to as photomechanics $[2,3,1]$. Photoelasticity was the first technique that was used to study dynamic phenomena [4, 5, 6, 7], in particular prop-

Amine Bouterf, François Hild, Stéphane Roux

Laboratoire de Mécanique et Technologie (LMT)

ENS Paris-Saclay / CNRS / Université Paris-Saclay, 61 avenue du Président Wilson

94235 Cachan cedex, France, e-mail: firstname.lastname@1mt.ens-paris-saclay.fr

Pascal Forquin

Laboratoire 3SR, Université Grenoble Alpes, 1270 rue de la piscine, Domaine Universitaire

38400 Saint Martin d'Hères, France, e-mail: pascal.forquin@3sr-grenoble.fr 
agating cracks [8]. Moiré interferometry was then introduced [9, 10]. Although its practical use was more difficult than photoelasticity [11], some applications were shown in the 90s $[11,12,13]$. In contrast to photoelasticity, this technique as well as the following ones, deal with the actual material of interest and not a substitute chosen for its birefringence properties. Caustics proved to be very useful for estimating stress intensity factors and crack propagation velocities [14, 15, 16, 17]. Digital image correlation [18], high-speed photography of laser and white light speckles $[19,20,21]$ started being utilized to analyze dynamic tests in the 80 s. Interferometric holography [22] and shearography [23] were subsequently introduced.

In the following, the measurement technique that is nowadays becoming very popular, namely, digital image correlation and its extensions will be presented. In Section 2, the general principles of image correlation and stereocorrelation are introduced. These techniques are utilized very frequently to monitor dynamic experiments with high-speed cameras. In many cases, the displacement (and strain) fields are not the final data to be assessed. They are generally post-processed to analyze the deformation and failure of various materials subjected to dynamic loadings. Different case studies dealing with such analyses of the dynamic behavior of materials are discussed in Section 3.

\section{Digital image correlation}

Digital image correlation consists in measuring displacement (or velocity) fields by registering (or correlating) a series of digital images (i.e., a set of pixels whose gray level is known). The first step of this and related approaches is the acquisition of the pictures during the experiment by use of cameras. It requires good quality imaging systems. It is to be emphasized that image correlation is not specific to - but can be used with - high speed imaging. High speed imaging opens up a range of new possibilities to tackle dynamic or impact mechanical behavior, but the basic principle of DIC in itself is unchanged. It is however fair to add that the usually small image definition and its potentially high noise level have motivated specific developments such as spatiotemporal regularization, which will be discussed below. Figure 1 shows two experimental configurations that were used in some of the cases discussed herein with a single high-speed camera (FASTCAM APX RS, Photron, Japan). Powerful lights (Dedolight 400D, Dedotec, Germany) were utilized to monitor experiments performed with in-house Hopkinson bars or a high-speed tension/compression testing machine (Dytesys, France).

\subsection{Incremental DIC}

Digital pictures are represented by matrices of (pixel) position $\boldsymbol{x}$, which are taken at different instants of time $t$. Incremental DIC (or 2D-DIC) is the first image cor- 


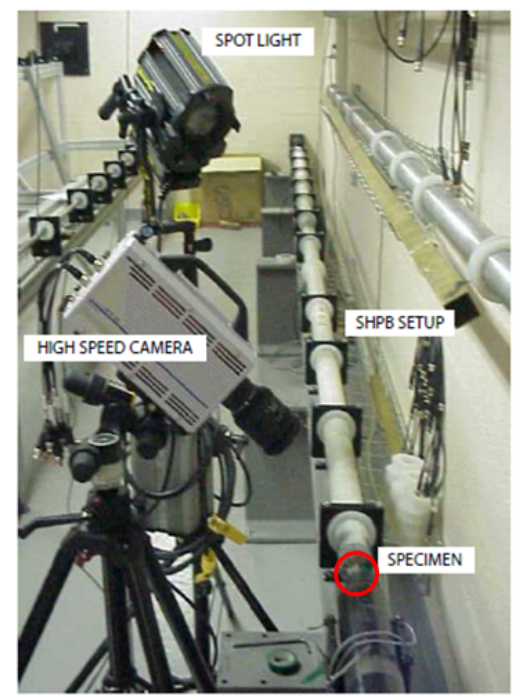

(a) taken from [24]

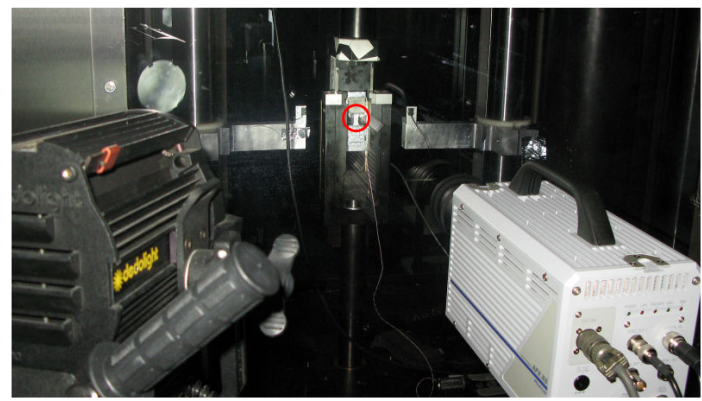

(b) courtesy of N. Swiergiel (Airbus Group Innovations)

Fig. 1 Two experimental configurations using one high-speed camera (FASTCAM APX RS, Photron, Japan) and powerful lighting (Dedolight 400D, Dedotec, Germany). The monitored sample is encircled in red

relation technique that was introduced in the early 1980s [25, 26, 27]. Only one camera (or imaging system) facing the region of interest is considered (Fig. 1) so that 2D displacements are measured. Its first use under dynamic loading conditions was reported in 1985 [18]. The stress intensity factor was evaluated and (favorably) compared to estimations with caustics. With the development of digital high-speed cameras, 2D-DIC has become popular for monitoring dynamic tests since the early 21 st century (see e.g., Refs. [28, 29, 24, 30, 31, 32, 33]).

The image of the observed surface in its reference configuration is denoted by $I_{0}$, and that of the surface in its deformed configuration at time $t$ by $I_{t}$. Figure 2 shows reference pictures $I_{0}$ of experiments to be discussed hereafter. The first one (Fig. 2(a)) corresponds to the natural texture of an aluminum foam tested in com- 


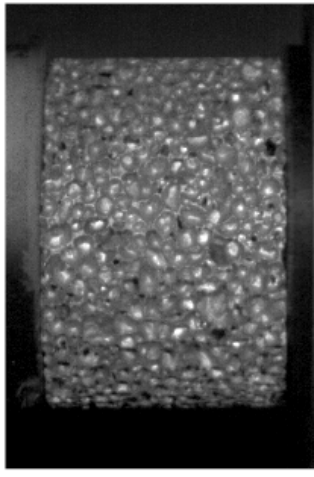

(a) Al foam

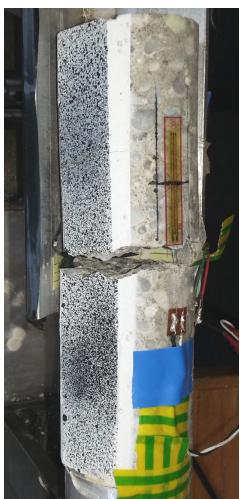

(b) concrete

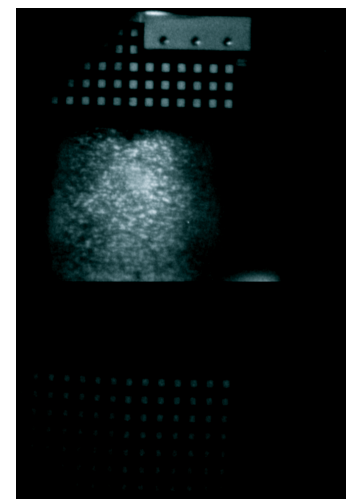

(c) copper cylinder

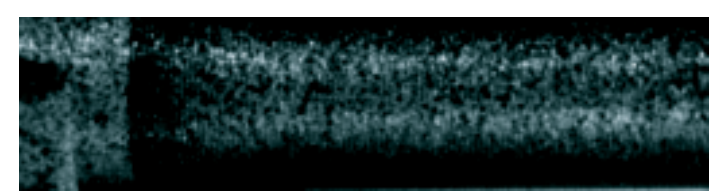

(d) aluminum

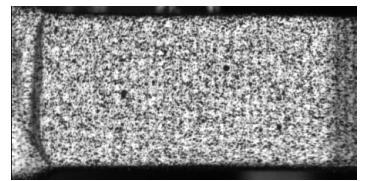

(e) composite

Fig. 2 Examples of pictures analyzed herein

pression (see Fig. 1(a)). Figure 2(b) shows a concrete sample fractured in dynamic spalling test. The random pattern was obtained by spraying a uniform white coating of paint and black dots. The third one (Fig. 2(c)) is one part of an image acquired during cylindrical expansion (Fig. 2(c)). In that case a sponge was used to deposit a very thin white speckle. Figure 2(d) shows the surface of a cylindrical sample to be tested in Hopkinson bars (Fig. 2(b)), which was sprayed with B/W paint to create a random pattern. Similarly, Fig. 2(e) shows the surface of a composite sample to be tested in tension.

Pictures $I_{0}$ and $I_{t}$ are related by

$$
I_{0}(\boldsymbol{x})=I_{t}(\boldsymbol{x}+\boldsymbol{u}(\boldsymbol{x}, t))+n(\boldsymbol{x}, t),
$$

where $\boldsymbol{u}(\boldsymbol{x}, t)$ is the displacement at pixel $\boldsymbol{x}$ and time $t$, and $n(\boldsymbol{x}, t)$ is a random component (e.g., photon noise, digitization when pictures are obtained by CCD or CMOS sensors, see Chapter 3). When $n=0$, Eq. (1) corresponds to the local brightness conservation (or gray level conservation). The determination of $\boldsymbol{u}$ is an ill-posed problem when no additional hypotheses are made. The information at hand (i.e., gray level of each pixel) does not allow for the evaluation of a vector field at the pixel scale. The registration is almost never performed down to the pixel level, but on a set of pixels referred to as zone of interest (ZOI), or on the region of interest (ROI) itself. Let us introduce the functional $\mathscr{R}$ of a trial displacement $\boldsymbol{u}$

$$
\mathscr{R}[\boldsymbol{u}]=\left\|I_{0}-I_{t}(\bullet+\boldsymbol{u})\right\|_{\Omega}^{2},
$$


where " $\bullet$ " denotes a dummy variable, $\Omega$ the considered domain where the registration will be performed (i.e., either any ZOI or the whole ROI). This setting corresponds to a weak formulation of the brightness conservation. When the usual $\mathscr{L}_{2}$ norm is considered, i.e., $\left\|I_{0}\right\|_{\Omega}^{2}=\sum_{\Omega}\left|I_{0}(\boldsymbol{x})\right|^{2}$, the determination of $\boldsymbol{u}$ consists in minimizing the sum of quadratic differences for, say, a rigid body translation [34]. The functional $\mathscr{R}_{c}$

$$
\mathscr{R}_{c}[\boldsymbol{u}]=\sum_{\Omega}\left[I_{0}(\boldsymbol{x})-I_{t}(\boldsymbol{x}+\boldsymbol{u}(\boldsymbol{x}, t))\right]^{2}
$$

is minimized with respect to the degrees of freedom of the sought displacement field. When the correct kinematics is found, and if $n=0$ [see Eq. (1)], $\mathscr{R}_{c}$ vanishes. Any offset will indicate that the registration was not perfect.

\subsubsection{Local approach}

Let us assume that $\boldsymbol{u}$ is a rigid body translation. The minimization of Eq. (3) can be solved with correlation techniques that consist in maximizing the superposition of shifted copies of $I_{t}$ and $I_{0}[25,26,27]$. The previous minimization is equivalent to maximizing (in the limit of large ZOIs) the quantity $c(\boldsymbol{u})$

$$
c(\boldsymbol{u})=\left(I_{0} \star I_{t}\right)(\boldsymbol{u}) \equiv \sum_{\mathrm{ZOI}} I_{0}(\boldsymbol{x}) I_{t}(\boldsymbol{x}+\boldsymbol{u})
$$

where $\star$ denotes the cross-correlation product. The value that maximizes the crosscorrelation product is an estimate of the unknown translation vector. The evaluation of the correlation product can be performed either in the reference space [27, 35] or Fourier space $[36,37,38]$. The measured field is composed of a set of displacements assigned to the center of all analyzed ZOIs.

Let us note that the hypothesis made so far (i.e., piece-wise constant displacements) can be relaxed. Nowadays academic and commercial correlation codes use local displacement interpolations of degree one or higher. However, only the mean value is kept and assigned to the center of each ZOI. It allows more complex features to be better captured, and leads to uncertainties whose amplitude depends, among others, on the texture and its gray level interpolation, but also on the type of local interpolation, and on the ZOI size [39, 47].

\subsubsection{Global approach}

The space of trial displacements $\mathscr{E}_{M}$ is introduced and satisfies the continuity requirement usually valid in experiments. The sought displacement is written as a linear combination in a basis of $\mathscr{E}_{M}$, namely, $\boldsymbol{u}(\boldsymbol{x}, t)=\sum_{i} v_{i}(t) \boldsymbol{\Psi}_{i}(\boldsymbol{x})$. The functional to minimize now reads

$$
\mathscr{R}_{c}[\{\boldsymbol{v}\}]=\sum_{\text {ROI }}\left[I_{0}(\boldsymbol{x})-I_{t}\left(\boldsymbol{x}+v_{i}(t) \boldsymbol{\Psi}_{i}(\boldsymbol{x})\right)\right]^{2}
$$


where $\{\boldsymbol{v}\}$ is the column vector that gathers all unknown amplitudes $v_{i}(t)$. Moreover, the displacement field is generally evaluated by successive iterations and corrections [40]. Let us assume that $I_{0}$ and $I_{t}$ be sufficiently regular at the smallest scales, and that the displacement corrections $\delta \boldsymbol{u}$ be small so that a first order Taylor expansion is possible, and $\mathscr{R}_{c}$ is a quadratic form of the unknown displacement corrections

$$
\mathscr{R}_{c}[\{\delta \boldsymbol{v}\}]=\sum_{\text {ROI }}\left[I_{0}(\boldsymbol{x})-\tilde{I}_{t}(\boldsymbol{x})-\delta v_{i}(t) \boldsymbol{\Psi}_{i}(\boldsymbol{x}) \cdot \boldsymbol{\nabla} I_{0}(\boldsymbol{x})\right]^{2}
$$

where $\tilde{I}_{t}$ denotes the corrected deformed picture (i.e., $\tilde{I}_{t}(\boldsymbol{x})=I_{t}\left(\boldsymbol{x}+u^{(n)}(\boldsymbol{x}, t)\right)$ for the current evaluation of the displacement field at iteration $n)$, and $\boldsymbol{\nabla} I_{t}\left(\boldsymbol{x}+u^{(n)}(\boldsymbol{x}, t)\right) \approx$ $\left.\nabla I_{0}(\boldsymbol{x})\right)$. The extremality condition for each amplitude $v_{i}(t)$ reads

$$
\left[\sum_{\mathrm{ROI}}\left(\nabla I_{0} \cdot \Psi_{i}\right)(\boldsymbol{x})\left(\boldsymbol{\nabla} I_{0} \cdot \boldsymbol{\Psi}_{k}\right)(\boldsymbol{x})\right] v_{k}(t)=\sum_{\mathrm{ROI}}\left(I_{0}-\tilde{I}_{t}\right)(\boldsymbol{x})\left(\boldsymbol{\nabla} I_{0} \cdot \boldsymbol{\Psi}_{i}\right)(\boldsymbol{x})
$$

This linear system can be written in matrix-vector form

$$
M_{i k} v_{k}(t)=a_{i}
$$

The regularity condition, which seems restrictive, can still allow non differentiable textures to be analyzed within the present framework [41]; the regularity of $\boldsymbol{u}$ allows one to bypass in many cases that of $I_{0}$ by integrations by parts and filtering. By implementing multiscale approaches to address the issue of large displacements and strains, the high frequency displacement components are progressively introduced [38, 42].

At this level of generality, various measurement spaces $\mathscr{E}_{M}$ can be chosen. This is one of the advantages of the present framework. For example, Fourier decompositions of the displacement field $[43,41]$ were considered to capture displacement fluctuations. A very often used alternative in solid mechanics is to discretize displacement fields with finite elements. The simplest choice when dealing with pictures is to consider 4-noded quadrilaterals with bilinear displacement interpolation (i.e., Q4-DIC [42]). More recently, unstructured meshes made of 3-noded triangles have also been utilized [44, 45].

\subsubsection{Uncertainty quantification}

One key aspect associated with kinematic measurements is related to the estimation of displacement uncertainties [39]. In particular, the hardware associated with high-speed cameras plays a significant role in the overall performance of such equipment [46]. Figure 3 compares the measurement uncertainty for three different highspeed and ultra high-speed cameras with different Q4 element sizes. This analysis was performed by acquiring a series of pictures in the reference configuration prior to an actual experiment. All the experimental conditions (e.g., acquisition pa- 
rameters, lighting (Dedocool COOLH, Dedotec, Germany)) were identical to the subsequent test. This series of images was analyzed via Q4-DIC and the standard displacement uncertainty was obtained.

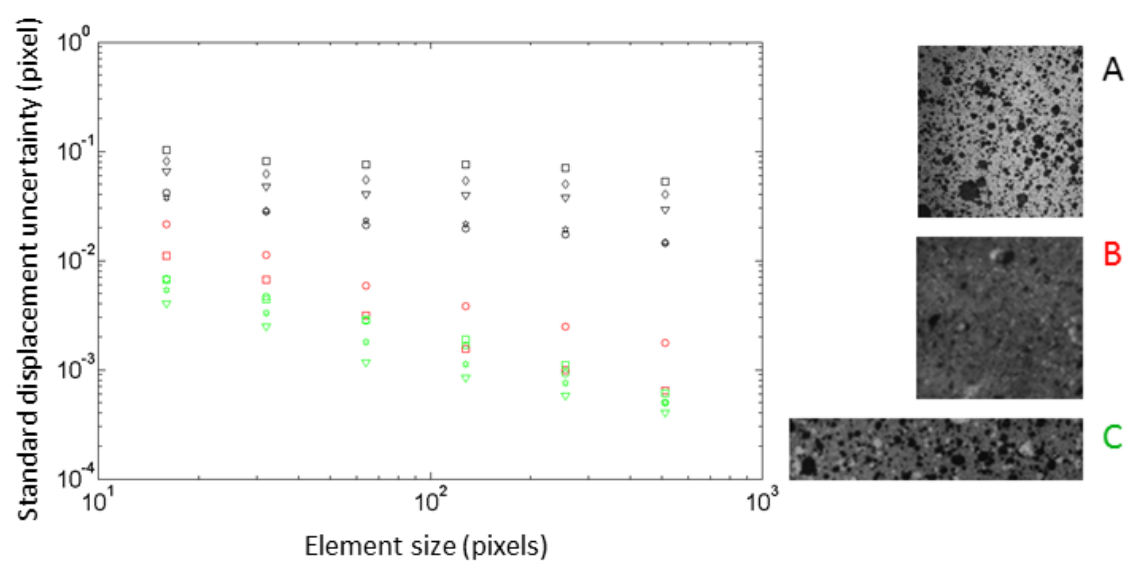

Fig. 3 Uncertainty quantification for three different high-speed cameras. For a given camera, the highest points correspond to a higher number of frames per second. Reference picture $I_{0}$ shot by the three analyzed cameras are also shown

The larger the element size, the lower the measurement uncertainty, which is expected. However, the three cameras do not yield the same type of result. Two cameras (i.e., B and C) lead to standard displacement uncertainties that are mostly controlled by acquisition noise [47]

$$
\sigma_{u}=\frac{\sqrt{6} \kappa \sigma_{I} p}{G_{I} \ell}
$$

where $\kappa$ is a constant of the order of 1 that depends whether it is an inner node, edge node or corner note, $p$ denotes the physical size of one pixel, and $G_{I}$ the root mean square gradient of the reference picture $I_{0}$. It is believed that camera A hardware leads to displacement fields that do not have a random signature associated with acquisition noise. Last, it is worth noting that for any camera, the higher the acquisition rate, the higher the measurement uncertainty (i.e., the lower the signal to noise ratio).

\subsection{Spatiotemporal DIC}

Virtually all applications of DIC for the investigation of dynamic experiments have not required specific algorithmic developments in comparison with quasi static tests. However, many high-speed cameras lead to image definitions that become very 
small as the number of frames per second increases. To compensate for this decrease in spatial definition, spatiotemporal algorithms have been introduced [48, 49] in which movies are analyzed.

An additional way of regularizing correlation procedures is to analyze a series of pictures (instead of an image pair in the previous section). This approach is referred to as spatiotemporal correlation (or 2D + t DIC) since the displacement field is now evaluated as a space / time decomposition $\boldsymbol{u}(\boldsymbol{x}, t)=\sum_{i} \sum_{j} v_{i j} \boldsymbol{\Psi}_{i}(\boldsymbol{x}) \phi_{j}(t)$. The sum of squared differences

$$
\mathscr{R}_{c}[\{\boldsymbol{v}\}]=\sum_{\left[t_{0}, t_{\max }\right]} \sum_{\mathrm{ROI}}\left[I_{0}(\boldsymbol{x})-I_{t}\left(\boldsymbol{x}+v_{i j} \boldsymbol{\Psi}_{i}(\boldsymbol{x}) \phi_{j}(t)\right)\right]^{2}
$$

is minimized with respect to the unknown degrees of freedom $v_{i j}$ over the ROI and time interval $\left[t_{0}, t_{\max }\right]$. The time discretization may consist of linear temporal elements extending over $n$ frames so that a spatiotemporal analysis considers 'volumes' of size $\ell^{2} n$ (expressed in voxels by equivalence with volume correlation) when the spatial discretization is based upon 4-noded quadrilaterals of edge size $\ell$.

To illustrate the gain in terms of displacement uncertainty, a picture (Fig. 2(e)) acquired with a high-speed camera (FASTCAM APX RS, Photron, Japan) is considered. Its definition is rather small (i.e., $224 \times 449$ pixels) and the analyzed ROI has a definition of $175 \times 336$ pixels. In the following a purely artificial uncertainty analysis is performed. It consists of adding to the picture of Fig. 2(e) a Gaussian white noise (zero mean, standard deviation $\sigma_{I}$ equal to $3 \%$ of the dynamic range) for each picture of the artificial movie. This level of noise is quite high for illustration purposes and also to check whether a priori predictions of the standard displacement uncertainty [49] are still valid for a series of $n$ pictures per time step in a single analysis

$$
\sigma_{u}=\frac{\sqrt{2} \sigma_{I} p}{G_{I} \ell} \sqrt{\alpha+\frac{\beta}{n}}
$$

where $\alpha+\beta$ is of the order of 3 , and the lower $\alpha$ the higher the gain when considering a spatiotemporal analysis.

Figure 4 shows a comparison between the standard displacement uncertainty $\sigma_{u}$ achieved with incremental $(n=1)$ and spatiotemporal $(n>1)$ analyses. As expected from the previous result, the larger the number of pictures per time step (i.e., the larger the size of the spatiotemporal volume), the smaller the standard displacement uncertainty. The fact that it levels off for large spatiotemporal volumes is due to the first picture that is also corrupted by noise. In the present case, a $30 \%$ gain can be expected when large spatiotemporal volumes are considered when compared with an incremental analysis. 


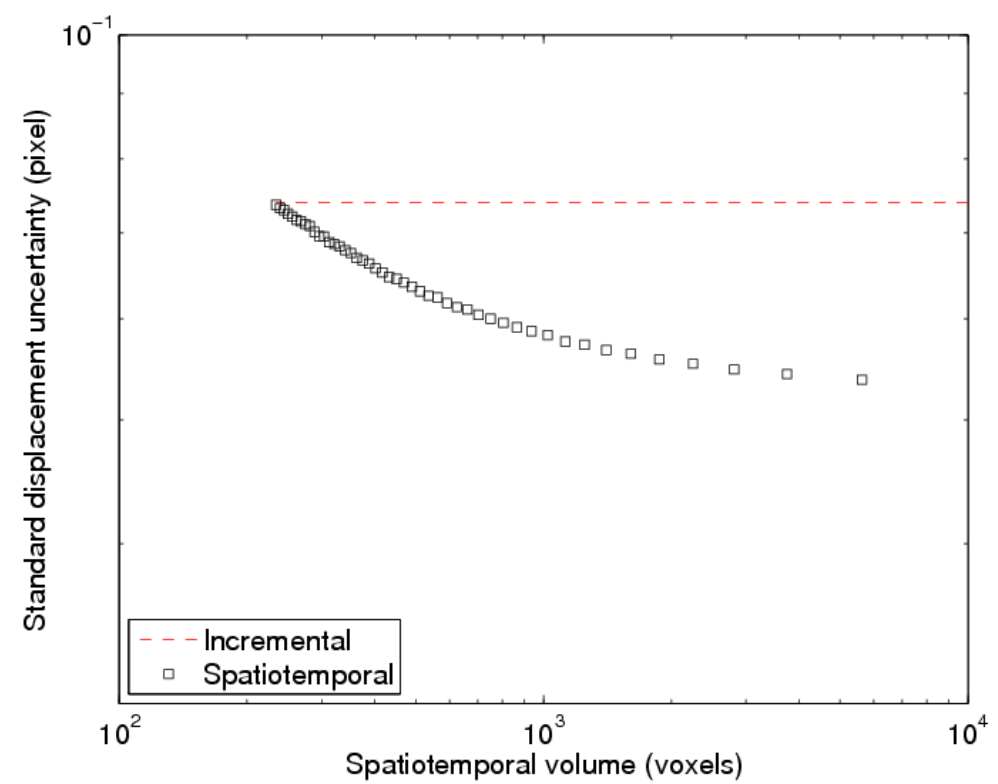

Fig. 4 Standard displacement uncertainty $\sigma_{u}$ as a function of the spatiotemporal volume. Comparison between incremental and spatiotemporal approaches

\subsection{Stereocorrelation}

Stereovision [50,35] consists in reconstructing 3D surfaces. By observing the latter from at least two different perspectives it is possible to determine the 3D coordinates of points. Once the initial 3D surface has been reconstructed, its 3D deformation can be measured during an experiment (i.e., 3D surface displacements). A calibrated stereoscopic setup (generally comprising two cameras, see Fig. 5(b)) and a DIC technique are used to register spatial and temporal sequences. This technique is widely used to monitor mechanical tests [53] for which monovision is not sufficient (because, for example, of out-of-plane motions [54]). Stereocorrelation can also be used to monitor ultra high-speed experiments (Fig. 5) as will be illustrated in the next section, provided the imaging devices are properly synchronized and characterized from a metrological standpoint $[55,46,56,51]$. It is also possible to use a single camera and two mirrors to acquire on picture two view points of the sample or structure [57, 51] (Fig. 5(a)). The second half of the last decade has seen the first applications of stereocorrelation to the analysis of high-speed experiments $[55,46,57,56]$. 


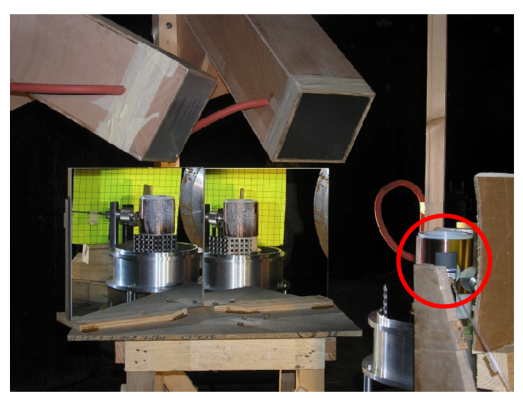

(a) taken from [51]

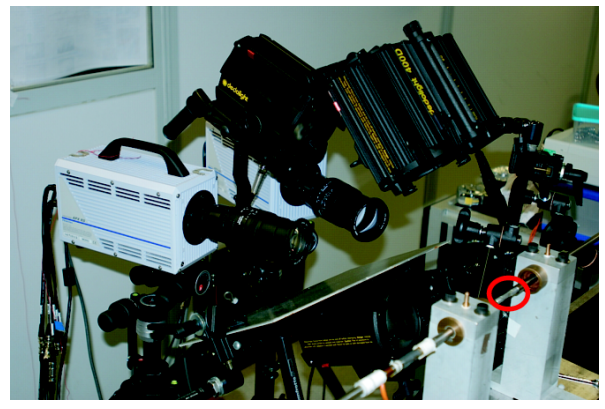

(b) taken from [52]

Fig. 5 Experimental configurations using either (a) one rotating mirror framing camera (LCA, France) or (b) two FASTCAM APX RS (Photron, Japan) cameras for stereocorrelation analyses. The monitored sample is encircled in red

\section{Applications}

\subsection{Shock enhancement of cellular materials}

The analysis of the deformation of different foams under shock loading is possible thanks to $2 \mathrm{D}-\mathrm{DIC}$.
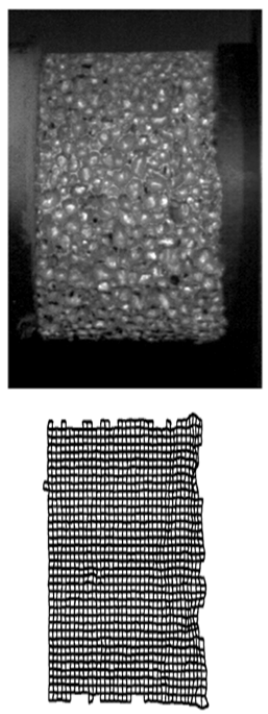

(a)
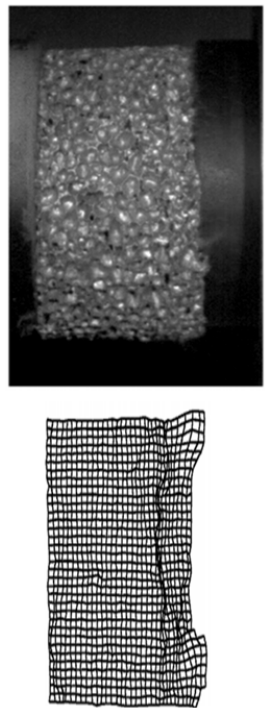

(b)
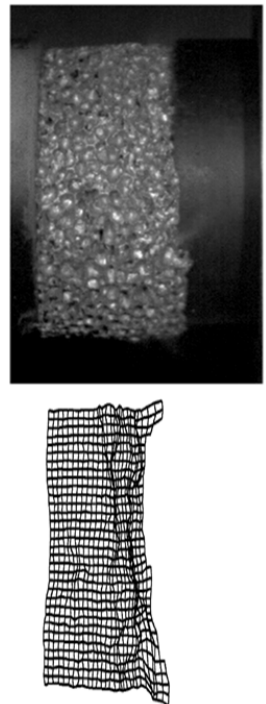

(c)

Fig. 6 Pictures of the deformed configuration (the reference configuration is shown in Fig. 2(a)) and corresponding mesh of Q4-DIC analyses [24] 
An original testing procedure using a large diameter Nylon Hopkinson bar is introduced. Tests under two different configurations (i.e., pressure bar behind/ahead of the assumed shock front) at the same shock speed are used to obtain the force/time histories behind and ahead of the shock front within cellular material samples [24]. Figure 1(a) shows one of the experimental configurations monitored by a highspeed camera (FASTCAM APX RS, Photron, Japan) for a tested aluminum foam (Fig. 2(a)). One of the two Dedocool 400D (Dedotec, Germany) lighting systems is also shown. A shock front is clearly revealed in the Q4-DIC analysis shown in Fig. 6 for a rather small impact velocity (here of the order of $50 \mathrm{~m} / \mathrm{s}$ ). From such type of data it could be shown that the experimentally observed shock enhancement was not due to the foam behavior but to a structural effect [58].

\subsection{Dynamic tensile damage and cracking of geomaterials}

For the last two decades, spalling techniques have been widely employed for investigating the tensile strength of geomaterials (e.g., standard concrete, high strength concrete, mortars, rocks) at strain rates ranging from $20 \mathrm{~s}^{-1}$ to $200 \mathrm{~s}^{-1}[59,60,61$, 62]. The experimental setup consists of using a single Hopkinson pressure bar (i.e., input bar) that is placed in contact with the tested sample. A short compressive pulse (blue dashed line, Fig. 7(a)), which is generated by a small explosive charge or the impact of a short projectile, propagates through the Hopkinson bar and the sample and reflects back at its free end as a tensile pulse (red dashed line, Fig. 7(a)). When the reflected pulse exceeds in amplitude the incident compressive pulse, a tensile loading is applied to the sample and causes its failure. 


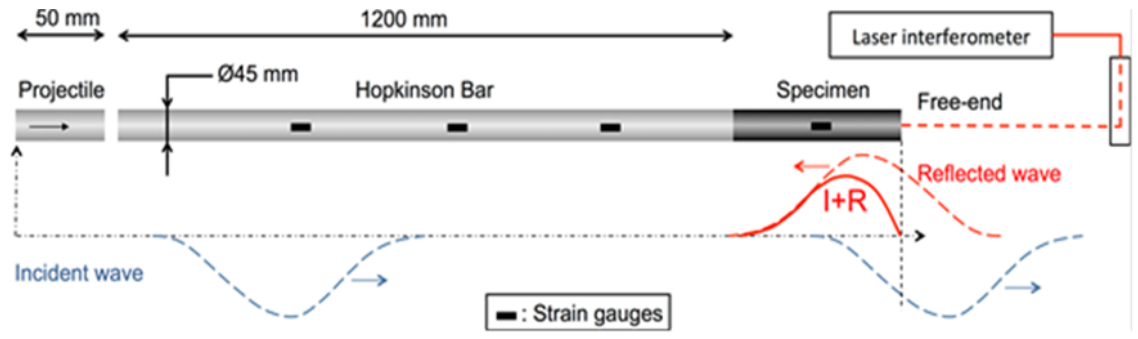

(a)

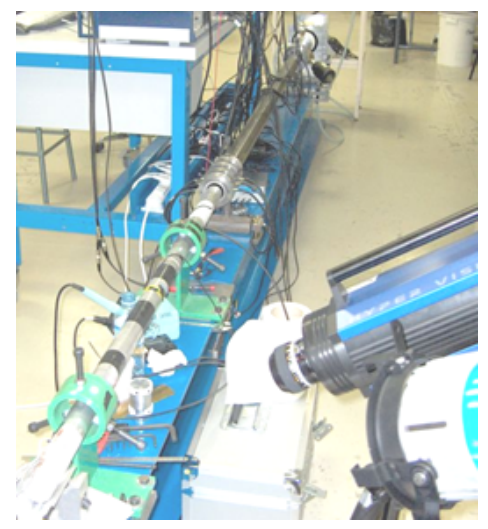

(b)

Fig. 7 (a) Principle of the spalling test. (b) View of the experimental setup [62]

Among different techniques used to identify the ultimate tensile strength of the sample during a spalling test the so-called Novikov method prevails [63, 60, 62]. However this method, which is based on the measurement of the velocity profile on the rear face of the sample, relies on the assumption of a linear-elastic behavior of the tested material until the peak-stress and only provides its spall strength with no information on its softening response. More recently, a new processing technique has been proposed [64] to evaluate the stress level in the sample and its apparent Young's modulus thanks to an ultra-high speed camera (HPV-1, Shimadzu, Japan) that is used to visualize the lateral surface of the sample (Fig. 7(b)). 2D displacement fields are obtained through full-field measurement techniques and DIC processing methods. The acceleration map is obtained by a double differentiation of the displacement field and a temporal fit of a second-order polynomial function over five images. Next, the mean stress in any cross-section along the length of the sample shot by the camera can be calculated. Knowing the strain level in the same crosssection, the stress-strain curve in any cross-section is obtained. The quality of the stress and strain measurements was evaluated by considering an aluminium alloy sample [65] and Q4-DIC [42].

The spalling technique provides a estimation of the tensile strength of concrete. However the tensile damage generated in the sample is composed of numerous ori- 
ented cracks that make their individual study and analysis very difficult. It is the reason why an experimental method referred to as "rocking spalling test" was proposed to investigate the dynamic propagation of a single crack in concrete samples under dynamic tensile loading [66]. The previous spalling setup is employed to apply a short compressive pulse to the sample. A parallelepiped is used instead of cylinder in which two notches are machined. The largest notch provides a "rocking" effect that produces a fast and local dynamic tensile loading in the zone near the tip of the small notch. The geometry of the specimen (size and location of notches) is shown in Fig. 8(a).

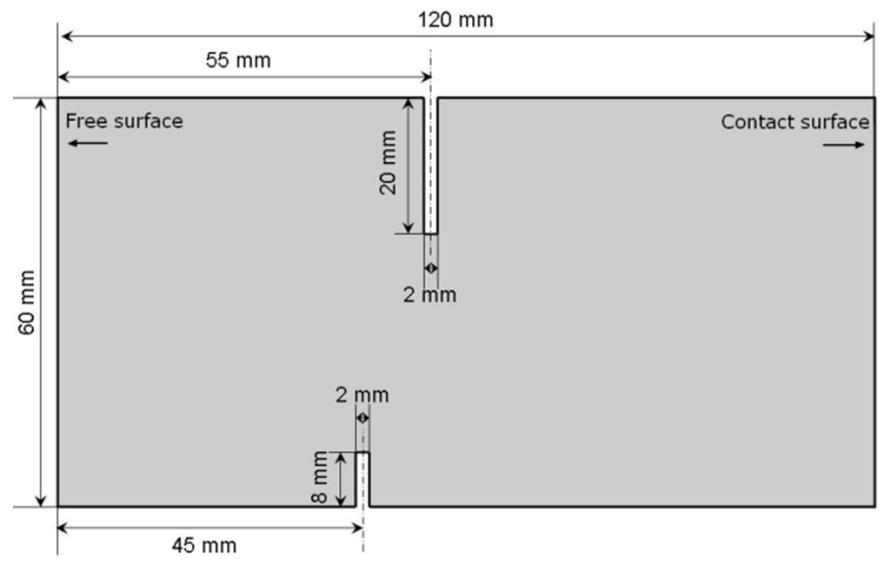

(a)

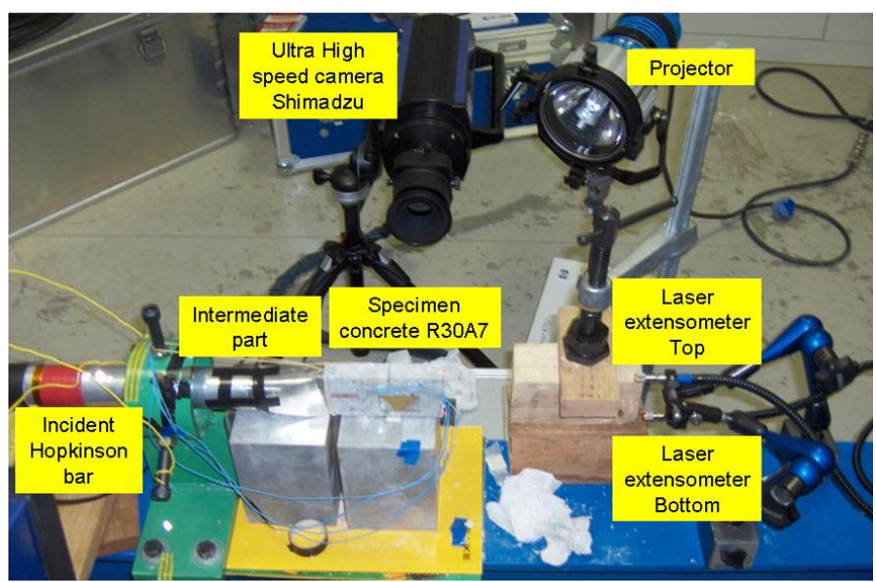

(b)

Fig. 8 (a) Specimen geometry used in rocking spalling tests. (b) Experimental setup used for dynamic crack propagation test [66] 
This configuration allows a single unstable crack to be initiated despite the high loading rate. Experiments were conducted on dry and water saturated concrete samples. The cracking inception and propagation can be visualized with an ultra-high speed digital camera and quantified via DIC. The results show that in most cases a single crack is indeed initiated on the small notch and propagates perpendicularly to the loading axis. Furthermore, the cracking velocity was evaluated with dry and water saturated concrete samples (i.e., $\approx 1300 \mathrm{~m} / \mathrm{s}[66])$.

\subsection{Tensile test on composite material}

The next example corresponds to an image sequence, which was acquired by a high-speed camera (FASTCAM APX RS, Photron, Japan) at 65,000 fps, of a tensile test (Fig. 1(b)) on a T700 carbon fiber / M21 epoxy matrix composite (the plies are oriented at $\pm 45^{\circ}$ with respect to the loading direction) with a high-speed tension/compression testing machine (Dytesys, France). The picture definition is $224 \times 449$ pixels (Fig. 2(e)). This is a case where the spatiotemporal analysis is useful to enable for a small spatial resolution by enlarging the temporal resolution. The experiment is deemed difficult because cracks appear during the analyzed sequence. The hypothesis of displacement continuity in the spatial and temporal domains will thus be violated. A spatiotemporal analysis is run with Q4 spatial elements of equivalent length less than 5.4 pixels. This very small length can be used since the temporal axis is divided into 5 increments of 18 pictures each. With these parameters, the spatiotemporal volume of interest is of the order of 5.3 Mvoxels.

The global residuals are observed to be of the order of $3 \%$ of the dynamic range of the reference picture for the analyzed sequence. There is a first regime during which the mean correlation residual is of the order of $2.4 \%$. It corresponds to a situation where no cracks are observed (Fig. 9). The reason for the increase of the mean correlation residual in the second regime (i.e., after picture no. 60) is due to cracking. This last conclusion can be drawn from the analysis of the normalized residual fields shown in Fig. 9. Cracks are clearly visible for the residual field corresponding to picture no. 53. Multiple cracking zones are observed in the residual of picture no. 71. Two fully damaged zones are seen in the residual field of picture no. 99. For the same pictures, the longitudinal displacement fields are also shown in Fig. 9. Multiple cracks are observed in the maps corresponding to picture no. 53. Their number increases and a lot of them are present in the maps corresponding to picture no. 99 . 

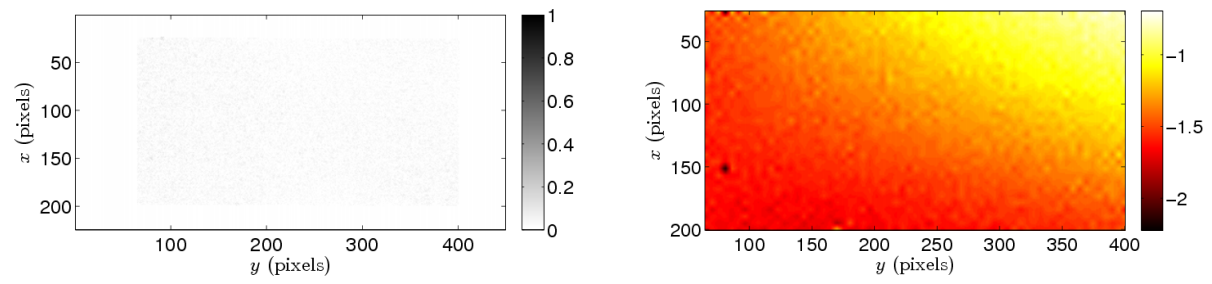

(a) picture no. 27
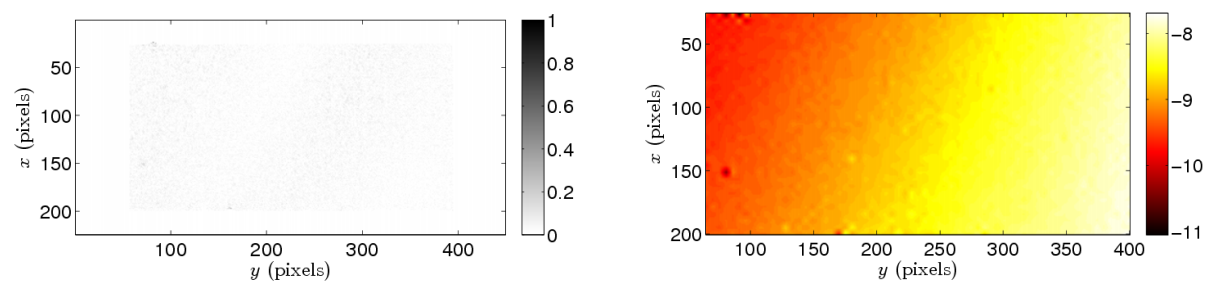

(b) picture no. 35
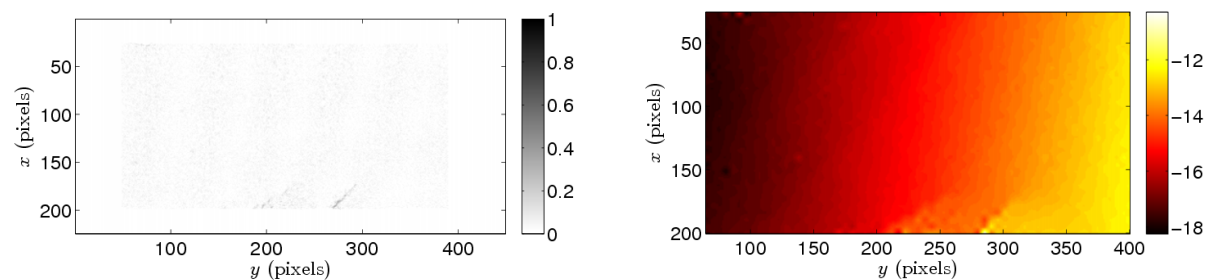

(c) picture no. 53
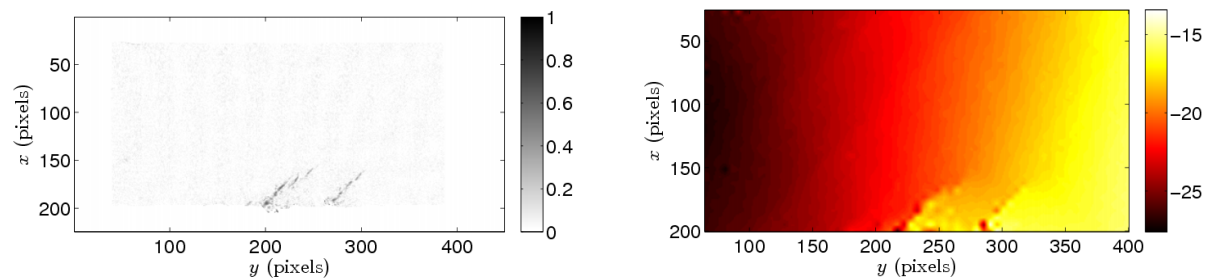

(d) picture no. 71
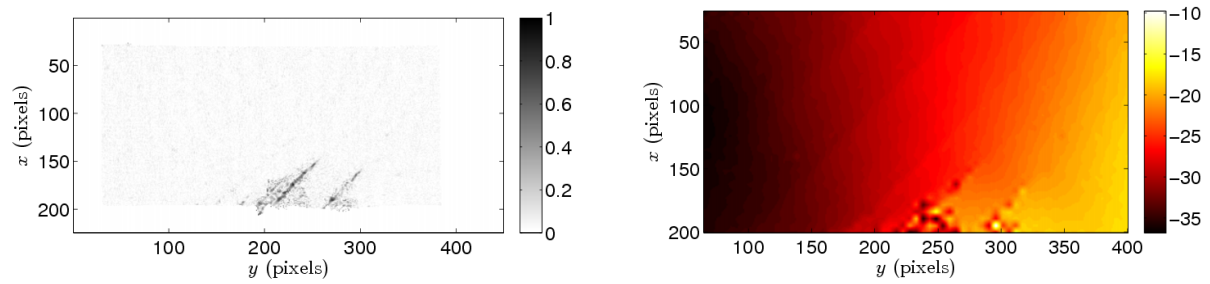

(e) picture no. 99

Fig. 9 Correlation residual (left) and horizontal (right) displacement maps (in pixels) at the end of the five time increments 


\subsection{Analysis of necking}

When axisymmetric samples are tested the use of stereocorrelation is desirable since out-of-plane motions generally occur. One counterexample was discussed in Section 3.1, namely, the foam Poisson's ratio was close to zero so that very small out-of-plane motions were expected, thereby making 2D-DIC approaches possible. When stereocorrelation techniques are chosen with two (or more) high-speed cameras (i.e., FASTCAM APX RS, Photron, Japan, in the present case), one key point is to ensure the synchronization of the stereovision setup (Fig. 5(b)). The latter was used to study necking in aluminum cylinders tested in high-speed tension. To reach strain levels as high as $600 \%$, enhanced registration procedures had to be developed [52]. Figure 10 shows two reconstructed 3D surfaces at the end of the test. Necking induces very severe radius variations.

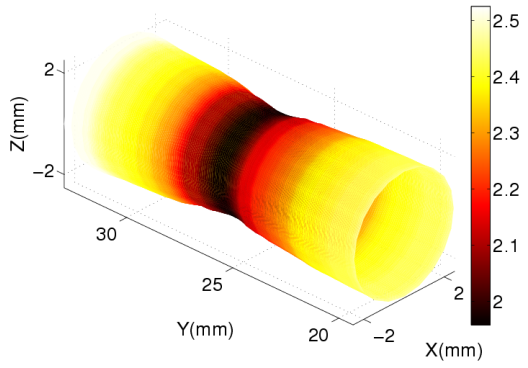

(a)

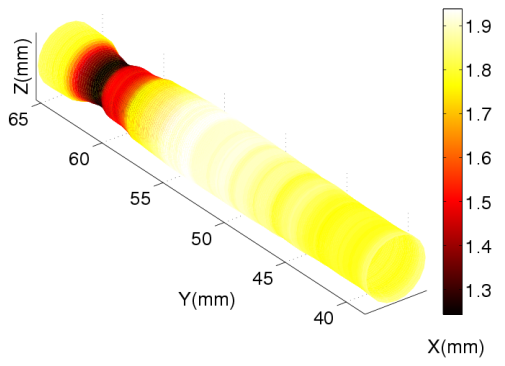

(b)

Fig. 10 Reconstructed 3D surfaces by enhanced stereocorrelation [52]. The color encodes the current radius of the sample tested in tensile Hopkinson bars

From such results, it was shown that the formation of the major neck was not strain rate sensitive up to levels equal to $1600 \mathrm{~s}^{-1}$. Conversely, the strain level at necking inception was observed to be random.

\subsection{Cylinder expansion}

This last example deals with a blast experiment in which a copper cylinder is subject to rapid expansion generated by an explosive. When following microsecond events it is impossible to synchronize two ultra high-speed cameras. Consequently, two mirrors and a single high-speed rotating mirror framing camera (LCA, France) and pyrotechnic flashes (CEA-DAM, France) are used to monitor the expansion (Fig. 5(a)). Further, calibration, which is usually carried out prior to the experiment [35], was 
not possible. Consequently two calibration targets are imaged during the experiment (Fig. 2(c)). Last, the registration is initialized with the displacement field predicted by hydrodynamic simulations of the experiment. Any offset between the measured and predicted displacement field is an indication of model error [51].

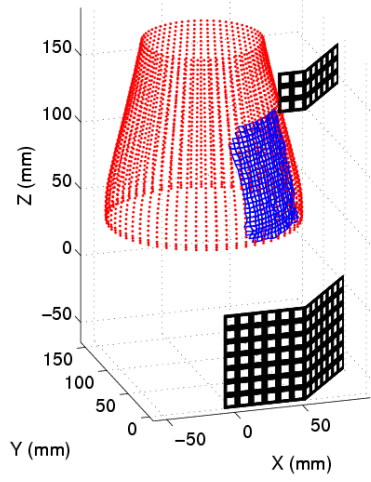

(a)

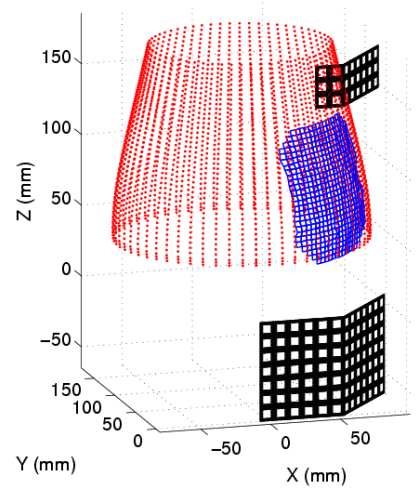

(b)

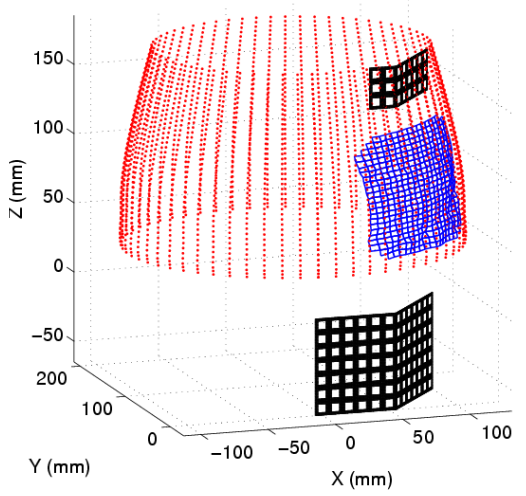

(c)

Fig. 11 3D shapes reconstructed via stereocorrelation. The blue mesh corresponds to the 3D reconstructed positions, and the red dots to the interpolated 3D surface

From a sequence of pictures it is possible to reconstruct the 3D shape of the cylinder at various instances of time (Fig. 11). For the more advanced shape, it is possible to observe the inception of necking. This result shows that even under such extreme conditions (i.e., very fast phenomena, lighting with pyrotechnic flashlights) it is possible to follow the deformation of surfaces up to very large strain levels (i.e., of the order of $100 \%$ ). 


\section{Conclusions}

With the development of new digital high-speed cameras with enhanced definition and higher acquisition rates, which become more affordable than in the past, their use in solid mechanics labs has increased during the last two decades. For example, one of the standard dynamic experiment (i.e., with Hopkinson bars) has been analyzed in more details and phenomena that could not be observed previously were revealed thanks to the measurement of kinematic fields via any photomechanical technique and in particular 2D-DIC or stereocorrelation techniques as above discussed. It is worth noting that one application, which was not discussed in the present chapter, is related to $4 \mathrm{D}$ tomography, which is also made possible thanks to nowadays high-speed cameras $[67,68]$. For example, the solidification of an aluminum alloy was monitored in a nondestructive way by such means [69]. Similarly, an in-situ tensile test on a metal matrix composite was studied with a frequency of 20 tomograms per second [70].

High-speed imaging allows deformation, damage and failure mechanisms to be analyzed in a qualitative and quantitative way. Such type of information is very useful when developing mechanism-based constitutive models. Further, DIC techniques allow quantitative estimates of kinematic fields to be performed. These sets of data can be compared with numerical simulations to validate the virtual models and/or calibrate parameters of constitutive models. Various identification techniques explicitly use full-field data [1]. These new approaches not only improve the way material parameters are calibrated, but they also enable the experimentalist to design new setups in which heterogeneity is no longer a limitation. Heterogeneous experiments can be imagined, not to make life more complicated, but to capture in a single test much more data so that the identification will be better conditioned.

Acknowledgements The results discussed herein have benefitted from the support of Agence Nationale de la Recherche (VULCOMP projects), CEA-DAM, CNRS and région Ile-de-France (SESAME project). The authors wish to thank G. Besnard, B. Durand, I. Elnasri, B. Erzar, Y. Girard, S. Guérard, J.-M. Guimard, J.-M. Lagrange, H. Leclerc, S. Pattofatto, D. Saletti, N. Swiergiel, $\mathrm{H}$. Tsitsiris and $\mathrm{H}$. Zhao for fruitful discussions.

\section{References}

1. M. Grédiac, F. Hild (eds.), Full-Field Measurements and Identification in Solid Mechanics (ISTE / Wiley, London (UK), 2012)

2. A. Lagarde, Advanced Optical Methods and Applications in Solid Mechanics, Solid Mechanics and its Applications, vol. 82 (Kluwer, Dordrecht (the Netherlands), 2000)

3. P. Rastogi (ed.), Photomechanics, Topics in Applied Physics, vol. 77 (Springer, Berlin (Germany), 2000)

4. A. Wells, D. Post, Proc. SESA 16, 69 (1958)

5. W. Bradley, A. Kobayashi, Exp. Mech. 10(3), 106 (1970)

6. J. Dally, Exp. Mech. 19(10), 349 (1979)

7. M. Ramulu, A. Kobayashi, D. Barker, Exp. Mech. 25(4), 344 (1985) 
8. A. Rosakis, O. Samudrala, D. Coker, Science 284(5418), 1337 (1999)

9. F. Chiang, G. Jaisingh, Exp. Mech. 13(4), 168 (1973)

10. J. Huntley, J. Field, Opt. Eng. 28(8), 926 (1989)

11. V. Deason, J. Epstein, M. Abdallah, Optics Lasers Eng. 12(2-3), 173 (1990)

12. K. Arakawa, J. Drinnon, R.H., M. Kosai, A. Kobayashi, Exp. Mech. 31(4), 306 (1991)

13. T. Bertin-Mourot, C. Denoual, G. Dehors, P. Louvigné, T. Thomas, J. Phys. IV Coll. C3(Suppl. III), 311 (1997)

14. J.F. Kalthoff, J. Beinert, S. Winkler, Measurements of Dynamic Sress Intensity Factors for Fast Running and Arresting Cracks in Double-Cantilever-Beam Specimens (1977), vol. STP 627

15. P. Theocaris, Int. J. Solids Struct. 14(8), 639 (1978)

16. K. Ravi-Chandar, W.G. Knauss, Int. J. Fract. 26(2), 141 (1984)

17. C. Liu, A. Rosakis, L. Freund, Int. J. Solids Struct. 30(7), 875 (1993)

18. W. Peters, W. Ranson, J. Kalthoff, S. Winkler, J. Phys. Coll. 46(C5), 631 (1985)

19. X. Hu, S. Palmer, J. Field, Optics Lasers Technol. 16(6), 303 (1984)

20. J. Huntley, S. Palmer, J. Field, Proc. SPIE 0814, 153 (1987)

21. B. Asay, G. Laabs, B. Henson, D. Funk, J. Appl. Phys. 82(3), 1093 (1997)

22. K.E. Fällström, H. Gustavsson, N.E. Molin, A. Wåhlin, Exp. Mech. 29(4), 378 (1989)

23. H. Tippur, A. Rosakis, Exp. Mech. 31(3), 243 (1991)

24. I. Elnasri, S. Pattofatto, H. Zhao, H. Tsitsiris, F. Hild, Y. Girard, J. Mech. Phys. Solids 55, 2652 (2007)

25. P. Burt, C. Yen, X. Xu, in IEEE Conf. on Pattern Recognition and Image Processing (IEEE, New York (NY), USA, 1982), pp. 269-274

26. W. Peters, W. Ranson, Opt. Eng. 21, 427 (1982)

27. M. Sutton, W. Wolters, W. Peters, W. Ranson, S. McNeill, Im. Vis. Comp. 1(3), 133 (1983)

28. F. Barthelat, Z. Wu, B. Prorok, H. Espinosa, Exp. Mech. 43(3), 331 (2003)

29. J. Kajberg, M. Sjödahl, in IUTAM Symposium on Field Analyses for Determination of Material Parameters - Experimental and Numerical Aspects, Solid Mechanics and its Applications, vol. 109, ed. by P. Ståhle, K. Sundin (Springer (the Netherlands), 2003), Solid Mechanics and its Applications, vol. 109, pp. 37-49

30. J. Kajberg, B. Wikman, Int. J. Solids Struct. 44(1), 145 (2007)

31. V. Tarigopula, O. Hopperstad, M. Langseth, A. Clausen, F. Hild, Int. J. Solids Struct. 45(2), $601(2008)$

32. F. Pierron, M. Sutton, V. Tiwari, Exp. Mech. 51(4), 537 (2011)

33. D. Saletti, S. Pattofatto, H. Zhao, Mech. Mat. 65, 1 (2013)

34. L. Gui, W. Merzkirch, Exps. in Fluids 28, 36 (2000)

35. M. Sutton, J. Orteu, H. Schreier, Image correlation for shape, motion and deformation measurements: Basic Concepts, Theory and Applications (Springer, New York, NY (USA), 2009)

36. D. Chen, F. Chiang, Y. Tan, H. Don, Appl. Optics 32, 1839 (1993)

37. F. Chiang, Q. Wang, F. Lehman, New Developments in Full-Field Strain Measurements Using Speckles (ASTM, Philadelphia (USA), 1997), vol. STP 1318, pp. 156-169

38. F. Hild, B. Raka, M. Baudequin, S. Roux, F. Cantelaube, Appl. Optics IP 41(32), 6815 (2002)

39. M. Bornert, F. Brémand, P. Doumalin, J. Dupré, M. Fazzini, M. Grédiac, F. Hild, S. Mistou, J. Molimard, J. Orteu, L. Robert, Y. Surrel, P. Vacher, B. Wattrisse, Exp. Mech. 49(3), 353 (2009)

40. F. Hild, S. Roux, Digital Image Correlation (Wiley-VCH, Weinheim (Germany), 2012), pp. $183-228$

41. B. Wagne, S. Roux, F. Hild, Eur. Phys. J. AP 17, 247 (2002)

42. G. Besnard, F. Hild, S. Roux, Exp. Mech. 46, 789 (2006)

43. S. Roux, F. Hild, Y. Berthaud, Appl. Optics 41(1), 108 (2002)

44. H. Leclerc, J. Périé, S. Roux, F. Hild, Integrated digital image correlation for the identification of mechanical properties (Springer, Berlin (Germany), 2009), vol. LNCS 5496, pp. 161-171

45. Z. Tomicevic, F. Hild, S. Roux, J. Strain Analysis 48, 330 (2013)

46. V. Tiwari, M. Sutton, S. McNeill, Exp. Mech. 47(4), 561 (2007)

47. F. Hild, S. Roux, Exp. Mech. 52(9), 1503 (2012)

48. G. Besnard, S. Guérard, S. Roux, F. Hild, Optics Lasers Eng. 49, 71 (2011) 
49. G. Besnard, H. Leclerc, S. Roux, F. Hild, J. Strain Analysis 47(4), 214 (2012)

50. P. Luo, Y. Chao, M. Sutton, W. Peters, Exp. Mech. 33, 123 (1993)

51. G. Besnard, J. Lagrange, F. Hild, S. Roux, C. Voltz, EURASIP J. Im. Video. Proc. 2010(215956), 15 p. (2010)

52. G. Besnard, F. Hild, J. Lagrange, P. Martinuzzi, S. Roux, Int. J. Impact Eng. 49, 179 (2012)

53. J. Orteu, Optics Lasers Eng. 47, 282 (2009)

54. M. Sutton, J. Yan, V. Tiwari, H. Schreier, J. Orteu, Optics Lasers Eng. 46(10), 746 (2008)

55. A. Gilat, T. Schmidt, J. Tyson, J. Phys. IV 134, 687 (2006)

56. P. Reu, T. Miller, J. Strain Anal. 43(8), 673 (2008)

57. G. Besnard, B. Etchessahar, J. Lagrange, C. Voltz, F. Hild, S. Roux, in 28th Int. Cong. HighSpeed Imaging and Photonics (Proc. SPIE 7126, 2008), p. 71261N (DOI:10.1117/12.821892)

58. S. Pattofatto, I. Elnasri, H. Zhao, H. Tsitsiris, F. Hild, Y. Girard, J. Mech. Phys. Solids 55, $2672(2007)$

59. J. Klepaczko, A. Brara, Int. J. Impact Eng. 25, 387 (2001)

60. H. Schuler, C. Mayrhofer, K. Thoma, Int. Journal Impact Eng. 32, 1635 (2006)

61. J. Weerheijm, J. van Doormaal, Int. J. Impact. Eng. 34, 609 (2007)

62. B. Erzar, P. Forquin, Exp. Mech. 50(7)

63. S. Novikov, I. Divnov, A. Ivanov, Fizika Metallov i Metallovedeniye 21, 608 (1966)

64. F. Pierron, M. Grédiac, The Virtual Fields Method (Springer, 2012)

65. D. Saletti, P. Forquin, Europ. Phys. J. Special Topics 225(2), 311 (2015)

66. P. Forquin, Eur. Phys. J. Spec. Topics 206(1), 89 (2012)

67. P. Lhuissier, M. Scheel, M. Di Michiel, E. Boller, J. Adrien, E. Maire, L. Salvo, J.J. Blandin, M. Suery, Ultra Fast Tomography: New Developments for 4D Studies in Material Science (John Wiley \& Sons, Inc., 2012), pp. 203-208

68. T. dos Santos Rolo, A. Ershov, T. van de Kamp, T. Baumbach, Proc. Nat. Acad. Sci. 111(11), $3921(2014)$

69. L. Salvo, M. Di Michiel, M. Scheel, P. Lhuissier, B. Mireux, M. Suéry, Ultra Fast In Situ XRay Micro-Tomography: Application to Solidification of Aluminium Alloys (Trans Tech Publications, 2012), vol. 706-709, pp. 1713-1718

70. E. Maire, C. Le Bourlot, J. Adrien, A. Mortensen, R. Mokso, Int. J. Fract. 200(1), 3 (2016) 